\title{
AN EXAMPLE OF ELLIPTIC STABILITY WITH LARGE PARAMETERS: LAME'S EQUATION AND THE ARNOLD-MOSER-RÜSSMANN CRITERION*
}

\author{
By \\ G. PECELLI ${ }^{1}$ AND E. S. THOMAS ${ }^{2}$
}

SUNY at Albany

1. Introduction. In this paper we consider two-degree-of-freedom Hamiltonian systems of the form:

$$
H=\frac{1}{2}\left(y_{1}{ }^{2}+y_{2}{ }^{2}\right)-a_{2} x_{1} x_{2}+\frac{1}{2}\left(a_{1}+a_{2}\right)\left(x_{1}{ }^{2}+x_{2}{ }^{2}\right)+\frac{\epsilon}{4}\left[b_{1}\left(x_{1}{ }^{4}+x_{2}^{4}\right)+b_{2}\left(x_{1}-x_{2}\right)^{4}\right]
$$

where $a_{1}, b_{1}>0 ; a_{2}, b_{2}, \epsilon \geq 0$.

For example, such a system may arise in nonlinear mechanics in the following way. One considers the idealized spring-mass system pictured in Fig. 1, where the outside springs are identical, both masses are equal to one, and the system is constrained to move horizontally. The displacements from equilibrium are denoted by $x_{1}$ and $x_{2}$, with $y_{i}=\dot{x}_{i}$. If the springs exert nonlinear restoring forces $F_{i}(\Delta)=a_{i} \Delta+\epsilon b_{i} \Delta^{3}$ corresponding to a displacement $\Delta$, then the equations of motion form a Hamiltonian system with the above integral. In what follows, it may be useful to keep this model in mind.

Associated with the system defined by (1) are two normal modes of vibration. The inphase mode is the periodic solution defined by $x_{1} \equiv x_{2}, y_{1} \equiv y_{2}$ and the out-of-phase mode is defined by $x_{1} \equiv-x_{2}, y_{1} \equiv-y_{2}$. We are interested in the iso-energetic stability of these modes; that is, we fix a total energy $h$ for the system and ask whether the given mode is orbitally stable in the energy surface $H=h$. This question has been treated by several authors $([3,8,9])$ under various assumptions, usually for weak nonlinearity (small $\epsilon$ ) or small energy. For example, in [3] it was shown that if the $a_{i}, b_{i}$ satisfy certain conditions, then the in-phase mode is iso-energetically stable for fixed $\epsilon$ and all sufficiently small $h$ or for fixed $h$ and all sufficiently small $\epsilon$. The methods used cannot give information about large values of $\epsilon h$. The purpose of this paper is to deal with stability for arbitrary values of these parameters.

For the in-phase mode our results are summarized in the following

THEOREM. Fix $a_{1}, a_{2}$ and $b_{2}$. There exist numbers $k_{1}$ and $k_{2}$ depending on $a_{1}, a_{2}$ and $b_{2}$, with $0<k_{1} \leq k_{2}<\infty$, such that if $\epsilon h$ lies in the set $D \equiv\left[0, k_{1}\right] \cup\left[k_{2}, \infty\right)$ then the inphase mode is elliptic or parabolic (in the surface $H=h)$ while if $\epsilon h$ lies in the set $H \equiv\left(k_{1}\right.$, $k_{2}$ ) it is hyperbolic (and hence unstable). Moreover, if $a_{2} \neq 0$, then there is a set $E$ of measure 0 in $D$ such that the in-phase mode is iso-energetically stable for all values of $\epsilon h$ in $D-E$.

* Received June 10, 1977.

${ }^{1}$ On leave from Hunter College, CUNY.

${ }^{2}$ Supported by the Alexander von Humboldt Foundation and the University of Würzburg. 


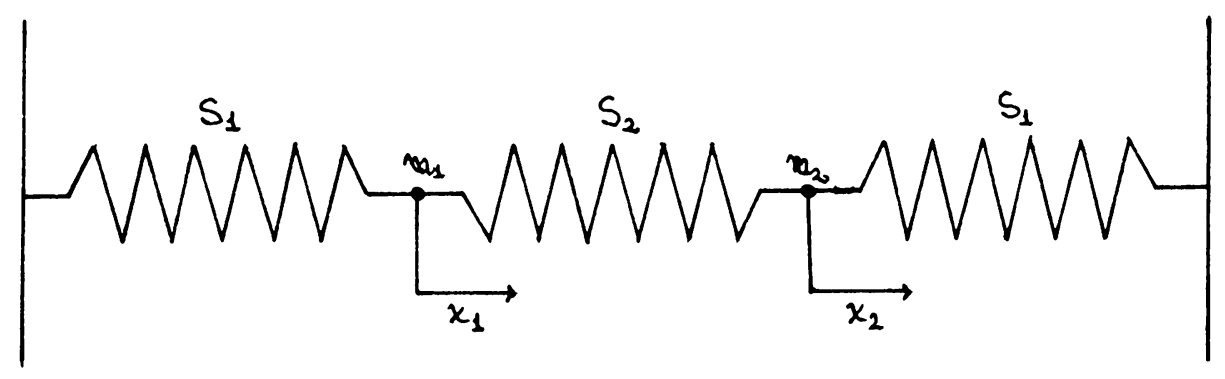

FIG. 1.

Thus, if $a_{2} \neq 0$, the mechanical system of Fig. 1 will be unstable for a bounded (possibly empty) set of values of $\epsilon h$ and on the complementary set will "almost always" be elliptic stable, even for strong nonlinearity and high energy.

Remarks. (1) The terms elliptic, parabolic and hyperbolic refer to the eigenvalues of the linearized Poincaré map (see Sec. 2) around the appropriate mode. In our context there are two eigenvalues, necessarily inverses, say $\Lambda$ and $\Lambda^{-1}$, and the mode is elliptic, parabolic or hyperbolic according as these have non-zero imaginary parts, are equal to $+1,+1$ or $-1,-1$, or are real and unequal, respectively.

(2) If $0 \leq a_{2} / a_{1} \leq 3 / 2$ then $k_{1}=k_{2}$, i.e., there is no hyperbolicity. If $a_{2} / a_{1}>3 / 2$ then $k_{1}$ $<k_{2}$ and these transitions to and from hyperbolicity are explicitly computed in Sec. 5.

The proof of the theorem depends on a study of the eigenvalues mentioned in remark (1) and on the Arnold-Moser-Rüssmann criterion which is based on the results of [1], [7], and $[10]$ and which we now summarize.

Let $H$ consist of those complex numbers $\mu$ of modulus one such that for some positive constants $c, \nu$ we have $\left|\mu^{n}-1\right|>\mathrm{cn}^{-\nu}$ for $n=1,2, \cdots$. The complement of $H$ has measure 0 in the unit circle (see Appendix 1).

Consider an area-preserving real analytic map $P$ of the form

$$
\begin{aligned}
x^{\prime} & =a x+b y+\cdots \\
P: & \\
y^{\prime} & =c x+d y+\cdots
\end{aligned}
$$

where the omitted terms represent power series beginning with terms of order at least 2 and converging in some neighborhood of the origin. Finally, suppose the eigenvalues of the linear part of $P$ are complex conjugate (therefore, since $P$ is area-preserving, necessarily of modulus 1 ).

Then, the origin is a stable fixed point of $\mathrm{P}$ provided the eigenvalues belong to $\mathrm{H}$.

The next four sections are devoted to the proof of the theorem. We reduce the variational equation to a Lamé equation and analyse the eigenvalues by means of a stability chart. (We remark that a similar technique was used in [4] to study periodic solutions of a different Hamiltonian system, the behavior of the eigenvalues, in that case, being governed by a Mathieu equation.) The out-of-phase solution, which behaves in a more complicated way and for which our techniques yield only partial results, is discussed in Sec. 6. We have included appropriate background material at various points to make the exposition more self-contained.

2. Reduction of the variational equation. We now fix a Hamiltonian of form (1) and consider the in-phase solution $\omega_{1}(t)$ defined by $x_{1} \equiv x_{2}, y_{1} \equiv y_{2}$. Denoting each of the two 
identical components by $(x(t), y(t))$, we see that $x$ and $y$ form a Hamiltonian system with Hamiltonian $\hat{H}$ given by

$$
\hat{H}=\frac{1}{2} y^{2}+\frac{a_{1}}{2} x^{2}+\frac{\epsilon b_{1}}{4} x^{4}
$$

If the total energy in the system is $h$, then the energy of each component is $h / 2$. The system corresponding to $\hat{H}$ can simply be written as Duffing's equation:

$$
\ddot{x}+a x+b x^{3}=0 ; \quad a=a_{1}, b=\epsilon b_{1} .
$$

The solution of (2) satisfying $x(0)=x_{0}, \dot{x}(0)=0$ may be written in terms of the Jacobi elliptic cosine [5]:

$$
\begin{gathered}
x=x_{0} \operatorname{cn}\left(u, \lambda^{2}\right) ; \\
t=\left(a+b x_{0}{ }^{2}\right)^{-1 / 2} u, \quad u=\int_{0}^{\phi}\left(1-\lambda^{2} \sin ^{2} \psi\right)^{-1 / 2} d \psi, \\
\lambda^{2}=\frac{1}{2} b x_{0}{ }^{2}\left(a+b x_{0}{ }^{2}\right)^{-1}, \quad c n\left(u, \lambda^{2}\right)=\cos \phi \text { (by definition). }
\end{gathered}
$$

In order to discuss the iso-energetic stability of $\omega_{1}(t)$ we need to compute the linearized Poincaré map of nearby solutions in the energy surface $H=h$. This is accomplished by computing the principal matrix solution $Z(t)$ of the associated variational equation. The rest of this section is concerned with expressing the variational equation in a suitable form.

Let

$$
K\left(\lambda^{2}\right)=\int_{0}^{\pi / 2}\left(1-\lambda^{2} \sin ^{2} \psi\right)^{-1 / 2} d \psi
$$

thus $c n\left(u, \lambda^{2}\right)$ has period $4 K\left(\lambda^{2}\right)$. Define $T\left(\lambda^{2}\right)$ by $T\left(\lambda^{2}\right)=2 K\left(\lambda^{2}\right)$.

We shall need the Hessian $H_{* *}$ of $H$ evaluated along $\omega_{1}(t)$. From (1) and the relations, $x_{1}=x_{2}=x, y_{1}=y_{2}=y$, one finds:

$$
H_{* *}\left(\omega_{1}\right)=\left[\begin{array}{cccc}
a_{1}+a_{2}+3 \epsilon b_{1} x^{2} & -a_{2} & 0 & 0 \\
-a_{2} & a_{1}+a_{2}+3 \epsilon b_{1} x^{2} & 0 & 0 \\
0 & 0 & 1 & 0 \\
0 & 0 & 0 & 1
\end{array}\right]
$$

(Here and below, we suppress the arguments of functions when no confusion can result.)

The variational equation around $\omega_{1}$ is the matrix equation

$$
\dot{z}=\left(\begin{array}{cc}
0 & I \\
-I & 0
\end{array}\right) H_{* *}\left(\omega_{1}\right) \mathrm{z}
$$

and we seek the principal matrix solution, i.e., the matrix $Z=\left(z_{i j}\right)$ such that $Z(0)=I_{4}$. Writing this equation in coordinates, we obtain:

$$
\begin{aligned}
& \dot{z}_{1 j}=z_{3 j}, \quad \dot{z}_{3 j}=-A z_{1 j}-B z_{2 j}, \\
& \dot{z}_{2 j}=z_{4 j}, \quad \dot{z}_{4 j}=-B z_{1 j}-A z_{2 j} ;
\end{aligned}
$$

or simply:

$$
\ddot{z}_{1 j}=-A z_{1 j}-B z_{2 j}, \ddot{z}_{2 j}=-B z_{1 j}-A z_{2 j}
$$


where $A=a_{1}+a_{2}+3 \epsilon b_{1} x^{2}, B=-a_{2}$.

To uncouple this system we introduce the variables

$$
u_{1 j}=z_{1 j}+z_{2 j}, u_{2 j}=z_{1 j}-z_{2 j} .
$$

Then the $u_{i j}$ satisfy:

$$
\begin{gathered}
\ddot{u}_{1 j}=-\left(a_{1}+3 \epsilon b_{1} x^{2}\right) u_{1 j}, \\
\ddot{u}_{2 j}=-\left(a_{1}+2 a_{2}+3 \epsilon b_{1} x^{2}\right) u_{2 j} .
\end{gathered}
$$

In view of the initial conditions

$$
\begin{aligned}
& u_{11}(0)=1, u_{12}(0)=1, u_{13}(0)=0, u_{14}(0)=0, \\
& \dot{u}_{11}(0)=0, \dot{u}_{12}(0)=0, \dot{u}_{13}(0)=1, \dot{u}_{14}(0)=1,
\end{aligned}
$$

we see that $u_{11} \equiv u_{12}$ and $u_{13} \equiv u_{14}$. In a similar way, $u_{22} \equiv-u_{21}$ and $u_{24} \equiv-u_{23}$.

We now transform Eqs. (4), (5) into Lamé equations as follows. By (3), $x=x_{0} c n\left(u, \lambda^{2}\right)$ with $u=\left(a+b x_{0}^{2}\right)^{1 / 2} t$. Using the identity $x^{2}=x_{0}{ }^{2}\left(1-\operatorname{sn}^{2}\left(u, \lambda^{2}\right)\right)$ Eq. (4) becomes:

$$
\ddot{u}_{1 j}=-\left(a_{1}+3 b_{1} x_{0}{ }^{2}-3 \epsilon b_{1} x_{0}{ }^{2} s n^{2}\left(u, \lambda^{2}\right)\right) u_{1 j} .
$$

Writing this in terms of the variable $u$ we have:

$$
\hat{u}_{1 j}^{\prime \prime}=-\left(\frac{a_{1}+3 \epsilon b_{1} x_{0}{ }^{2}}{a_{1}+\epsilon b_{1} x_{0}{ }^{2}}-\frac{3 \epsilon b_{1} x_{0}{ }^{2}}{a_{1}+\epsilon b_{1} x_{0}{ }^{2}} s n^{2}\left(u, \lambda^{2}\right)\right) \hat{u}_{1 j}
$$

where $\hat{u}_{1 j}(u)=u_{1 j}(t)$ and prime denotes differentiation with respect to $u$. Recalling from (3) that $\lambda^{2}=\frac{1}{2} \epsilon b_{1} x_{0}{ }^{2}\left(a_{1}+\epsilon b_{1} x_{0}{ }^{2}\right)^{-1}$, we finally obtain in place of (4) the equation

$$
\hat{u}_{1 j}^{\prime \prime}+\left(\left(4 \lambda^{2}+1\right)-2 \cdot 3 \lambda^{2} \operatorname{sn}^{2}\left(u, \lambda^{2}\right)\right) \hat{u}_{1 j}=0,
$$

with initial conditions:

$$
\begin{gathered}
\hat{u}_{1 j}(0)=1, \hat{u}_{1 j}{ }^{\prime}(0)=0, \quad j=1,2, \\
\hat{u}_{1 j}(0)=0, \hat{u}_{1 j}{ }^{\prime}(0)=\left(a+b x_{0}{ }^{2}\right)^{-1 / 2}, \quad j=3,4 .
\end{gathered}
$$

Similarly, Eq. (5) may be rewritten as:

$$
\hat{u}_{2 j}{ }^{\prime \prime}+\left(4 \lambda^{2}+1+2 a_{2}\left(a_{1}+\epsilon b_{1} x_{0}{ }^{2}\right)^{-1}-2 \cdot 3 \lambda^{2} s n^{2}\left(u, \lambda^{2}\right)\right) \hat{u}_{2 j}=0
$$

with initial conditions:

$$
\begin{gathered}
\hat{u}_{2 j}(0)=(-1)^{j-1}, \hat{u}_{2 j}{ }^{\prime}(0)=0 \text { for } j=1,2 \\
\hat{u}_{2 j}(0)=0, \hat{u}_{2 j}{ }^{\prime}(0)=(-1)^{j-1}\left(a+b x_{0}{ }^{2}\right)^{-1} \text { for } j=3,4 .
\end{gathered}
$$

We conclude this section by computing $x_{0}$ in terms of the other parameters of the problem. By the definition of $\hat{H}$ and the subsequent remark, we have:

$$
\epsilon b_{1} x_{0}{ }^{4}+2 a_{1} x_{0}{ }^{2}-2 h=0 .
$$

Solving for $x_{0}^{2}$ gives

$$
x_{0}^{2}=\left(a_{1}^{2}+2 h \epsilon b_{1}-a_{1}\right)^{1 / 2} / \epsilon b_{1}
$$

Then from (3) we obtain.

$$
\lambda^{2}=\frac{1}{2}-a_{1} / 2\left(a_{1}{ }^{2}+2 h \epsilon b_{1}\right)^{1 / 2} .
$$


We also have the useful relation

$$
\frac{2 a_{2}}{a_{1}+\epsilon b_{1} x_{0}{ }^{2}}=\frac{2 a_{2}}{\left(a_{1}{ }^{2}+2 h \epsilon b_{1}\right)^{1 / 2}} .
$$

3. The eigenvalues of $Z(\tau)$. We must now examine the eigenvalues of the linearized Poincare map followed through one full period $\tau$ of the in-phase solution.

Since the tangent vector along the orbit of $\omega_{1}(t)$ and the normal to the energy surface $H$ $=h$ are invariant under $Z(\tau)$, two of the eigenvalues are 1 . Furthermore, $\operatorname{det} Z(\tau)=1$ by the Hamiltonian character of the flow. Thus $Z(\tau)$ is a $4 \times 4$ matrix satisfying det $\mid Z(\tau)-$ $\mu I \mid=(\mu-1)^{2}\left(\mu^{2}+B \mu+1\right)$ for some constant $B$. The coefficient of $\mu^{3}$ on the left-hand side is $-\operatorname{Tr} Z(\tau)$ and in the right it is $B-2$. With this is mind we see that the two remaining eigenvalues of $Z(\tau)$ are:

$$
\Lambda_{ \pm}=\frac{1}{2}\left[D-2 \pm(D(D-4))^{1 / 2}\right],
$$

where $D=\operatorname{Tr} Z(\tau)$.

Our task is now to express $\operatorname{Tr} Z(\tau)$ in terms of the solutions $\hat{u}_{i j}$ of the Lamé equations (6) and (7). Using the relations between the $z_{i j}, u_{i j}$ and $\hat{u}_{i j}$ of the previous section, one finds:

$$
\begin{aligned}
\operatorname{Tr} Z(\tau) & =\sum z_{i i}(\tau) \\
& =\frac{1}{2}\left[\left(u_{11}+u_{21}\right)+\left(u_{12}-u_{22}\right)+\left(\dot{u}_{13}+\dot{u}_{23}\right)+\left(\dot{u}_{14}-\dot{u}_{24}\right)\right]_{t=\tau} \\
& =\left[\left(u_{11}+\dot{u}_{13}\right)+\left(u_{21}+\dot{u}_{23}\right)\right]_{t=\tau} \\
& =\left[\left(\hat{u}_{11}+\hat{u}_{13}{ }^{\prime}\right)+\left(\hat{u}_{21}+\hat{u}_{23}{ }^{\prime}\right)\right]_{u=2 T\left(\lambda^{2}\right)}
\end{aligned}
$$

where, with a slight abuse of notation, we have replaced the old $\hat{u}_{j 3}$ by multiples which have the initial values $\hat{u}_{j 3}(0)=0, \hat{u}_{j 3}{ }^{\prime}(0)=1$, the effect being to enable us to write $\hat{u}_{j 3}{ }^{\prime}\left(2 T\left(\lambda^{2}\right)\right)=\dot{u}_{j 3}(\tau)$. Here it may be useful to note that $\tau$, the " $t$-period" of $\omega_{1}$, is related to $2 T\left(\lambda^{2}\right)$, the " $u$-period" of $\omega_{1}$, by the formulas

$$
\tau=\left(a_{1}{ }^{2}+2 h \epsilon b_{1}\right)^{-1 / 2} 2 T\left(\lambda^{2}\right)=a_{1}{ }^{-1}\left(1-2 \lambda^{2}\right) 2 T\left(\lambda^{2}\right)
$$

which may be obtained using (3), (8), (9) and (10).

To further reduce the expression $\operatorname{Tr} Z(\tau)$ we use the following general result about Hill's equation with periodic coefficients.

LEMMA $([2,6])$. Let $y_{1}$ and $y_{2}$ be solutions of $y^{\prime \prime}+Q y=0$ where $Q$ is periodic with period $P$ and symmetric, $Q(u)=Q(-u)$. Moreover, suppose $y_{1}$ and $y_{2}$ are normalized: $y_{1}{ }^{\prime}(0)=1, y_{1}{ }^{\prime}(0)=0 \quad y_{2}(0)=0, y_{2}{ }^{\prime}(0)=1$. Then:

i) $y_{1}(u \pm P)=y_{1}(P) y_{1}(u) \pm y_{1}{ }^{\prime}(p) y_{2}(u)$;

ii) $y_{2}(y \pm P)= \pm y_{2}(P) y_{1}(u)+y_{2}^{\prime}(P) y_{2}(u)$;

iii) $y_{1}(u) y_{2}{ }^{\prime}(u)-y_{2}(u) y_{1}{ }^{\prime}(u) \equiv 1$;

iv) $y_{1}(P)=y_{2}^{\prime}(P)$.

Applying the lemma to the solutions $\hat{u}_{11}$ and $\hat{u}_{13}$ (which are now normalized) of Eq. (6), we may write:

$$
\begin{aligned}
\hat{u}_{11}(2 T)+\hat{u}_{13}{ }^{\prime}(2 T)= & \hat{u}_{11}{ }^{2}(T)+\hat{u}_{11}{ }^{\prime}(T) \hat{u}_{13}(T)+\hat{u}_{11}{ }^{\prime}(T) \hat{u}_{13}(T)+u_{13}{ }^{\prime 2}(T) \\
& =2 \hat{u}_{11}{ }^{2}(T)+2\left[\hat{u}_{11}(T) \hat{u}_{13}{ }^{\prime}(T)-1\right] \\
& =4 \hat{u}_{11}{ }^{2}(T)-2 .
\end{aligned}
$$


In a similar way, we have:

$$
\hat{u}_{21}(2 T)+\hat{u}_{23}{ }^{\prime}(2 T)=4 \hat{u}_{21}{ }^{2}(T)-2 .
$$

Substituting in our last expression for $\operatorname{Tr} Z(\tau)$ now yields:

$$
\operatorname{Tr} Z(\tau)=4\left[\hat{u}_{11}{ }^{2}(T)+\hat{u}_{21}{ }^{2}(T)-1\right] .
$$

Now it can easily be checked that the function $\operatorname{sn}(u) \cdot d n(u)$ satisfies Eq. (6) and has initial values coinciding with those of our modified $\hat{u}_{13}$. Hence

$$
\hat{u}_{11}(T)=\hat{u}_{13}{ }^{\prime}(T)=\mathrm{cn} T d n^{2} T-\lambda^{2} s n^{2} T d n T=-1 .
$$

Thus we finally obtain:

$$
\operatorname{Tr} Z(\tau)=4 \hat{u}_{21}{ }^{2}(T) .
$$

From this equation together with formula (11) we see that $0<\hat{u}_{21}{ }^{2}(T)<1$ implies the inphase solution is elliptic, $\hat{u}_{21}{ }^{2}(T)>1$ implies it is hyperbolic and $\hat{u}_{21}{ }^{2}(T)=0$ or 1 implies it is parabolic.

It remains to examine how the value of $\hat{u}_{21}(T)$ varies as a function of $\epsilon h$.

4. The stability chart of a special Lamé equation. Note that Eq. (7), which determines $\hat{u}_{21}$, is a special case of the Lamé equation

$$
y^{\prime \prime}+\left(\alpha-6 \lambda^{2} \operatorname{sn}\left(u, \lambda^{2}\right)\right) y=0 .
$$

By constructing the stability chart of (12) we shall be able to track the values of $\hat{u}_{21}$ as $\epsilon h$ varies from 0 to $\infty$, and ultimately to reach the main theorem.

To begin with, we summarize briefly some information about (12) or, more generally, about Hill's equations having the form

$$
y^{\prime \prime}+(\alpha+Q(u, \beta)) y=0
$$

where $\alpha$ and $\beta$ are real parameters, $Q$ is sufficiently smooth in $u$ and $\beta$ and periodic in $u$ with period $T$ depending on $\beta$. Modulo the extra parameter $\beta$ (which is present in our problem but does not affect what we are now going to say), this material can be found in [6]. The discriminant of Eq. (13) is the quantity $\Delta(\alpha, \beta)=y_{1}(T)+y_{2}{ }^{\prime}(T)$, where $y_{t}(u)=$ $y_{i}(u ; \alpha, \beta)$ are normalized solutions of (13); that is, they are the unique solutions satisfying $y_{1}(0)=1, y_{1}^{\prime}(0)=0, y_{2}(0)=0, y_{2}{ }^{\prime}(0)=1$. In case $Q$ is symmetric, one can, by the lemma quoted in the previous section, write the discriminant as $\Delta(\alpha, \beta)=2 y_{1}(T)$. Eq. (13) possesses solutions of period $T, 2 T$, respectively, precisely when $\Delta(\alpha, \beta)=2,-2$ respectively. The curves $\Delta(\alpha, \beta)= \pm 2$ divide the $\alpha, \beta$ plane into alternating so-called stable and unstable regions in which $\Delta^{2}<4, \Delta^{2}>4$ respectively. (The stability, of course, refers to (13) and not directly to our Poincaré map.) It can happen that instability regions collapse, leaving stability regions separated by a curve $\Delta= \pm 2$. The stability chart consists of the stable and unstable regions and the curves $\Delta= \pm 2$. Such charts exist for the Mathieu equation [5], but do not seem to have been constructed for the more difficult Lamé equation, particularly with the modulus of ellipticity taken into account.

Our Lamé equation (12) is of the form (13) with $\alpha=\alpha, \beta=\lambda^{2}, Q=-6 \lambda^{2} s n^{2}\left(u, \lambda^{2}\right)$. We begin by observing that (12) has the following periodic solutions [1, p. 205]:

$$
\begin{aligned}
y=1-\alpha / 2 \operatorname{sn}^{2}\left(u, \lambda^{2}\right) \text { when } \alpha=2\left(\left(1+\lambda^{2}\right)-\left(\lambda^{4}-\lambda^{2}+1\right)^{1 / 2}\right), & \text { period } T\left(\lambda^{2}\right), \\
y=c n\left(u, \lambda^{2}\right) \operatorname{dn}\left(u, \lambda^{2}\right) \text { when } \alpha=1+\lambda^{2}, & \text { period } 2 T\left(\lambda^{2}\right),
\end{aligned}
$$




$$
\begin{aligned}
y=\operatorname{sn}\left(u, \lambda^{2}\right) \operatorname{dn}\left(u, \lambda^{2}\right) \text { when } \alpha=1+4 \lambda^{2}, & \text { period } 2 T\left(\lambda^{2}\right), \\
y=\operatorname{sn}\left(u, \lambda^{2}\right) c n\left(u, \lambda^{2}\right) \text { when } \alpha=4+\lambda^{2}, & \text { period } T\left(\lambda^{2}\right), \\
y=1-\alpha / 2 s n^{2}\left(u, \lambda^{2}\right) \text { when } \alpha=2\left(\left(1+\lambda^{2}\right)+\left(\lambda^{4}-\lambda^{2}+1\right)^{1 / 2}\right), & \text { period } T\left(\lambda^{2}\right) .
\end{aligned}
$$

Thus each of the above curves $\alpha=\alpha\left(\lambda^{2}\right)$ is a boundary curve of a stability region (see Fig. 2). On the other hand, a Lamé equation of the form $y^{\prime \prime}+\left(\alpha-n(n+1) \lambda^{2} s n^{2}\left(u, \lambda^{2}\right)\right) y$ $=0$ has exactly $n+1$ nondegenerate instability regions (see [6, pp. 103, 106]). In our case, then, all instability intervals above the top curve collapse and we are left with Fig. 2.

Recall from Eq. (7) that we are interested in those equations of form (12) for which

$$
\alpha=4 \lambda^{2}+1+2 a_{2}\left(a_{1}+\epsilon b_{1} x_{0}{ }^{2}\right)^{-1},
$$

which with the aid of (10) can be written

$$
\alpha=4 \lambda^{2}\left(1-\frac{a_{2}}{a_{1}}\right)+2 \frac{a_{2}}{a_{1}}+1 .
$$

For various values of $a_{2} / a_{1}$ these form a family of lines which appear dotted in Fig. 2 .

Here one should note, by virtue of (9), that as $\epsilon h$ goes from 0 to $\infty, \lambda^{2}$ tends monotonically from 0 to $\frac{1}{2}$. Thus as $\epsilon h$ goes from 0 to $\infty$, the point $\left(\alpha, \lambda^{2}\right)$ moves on the

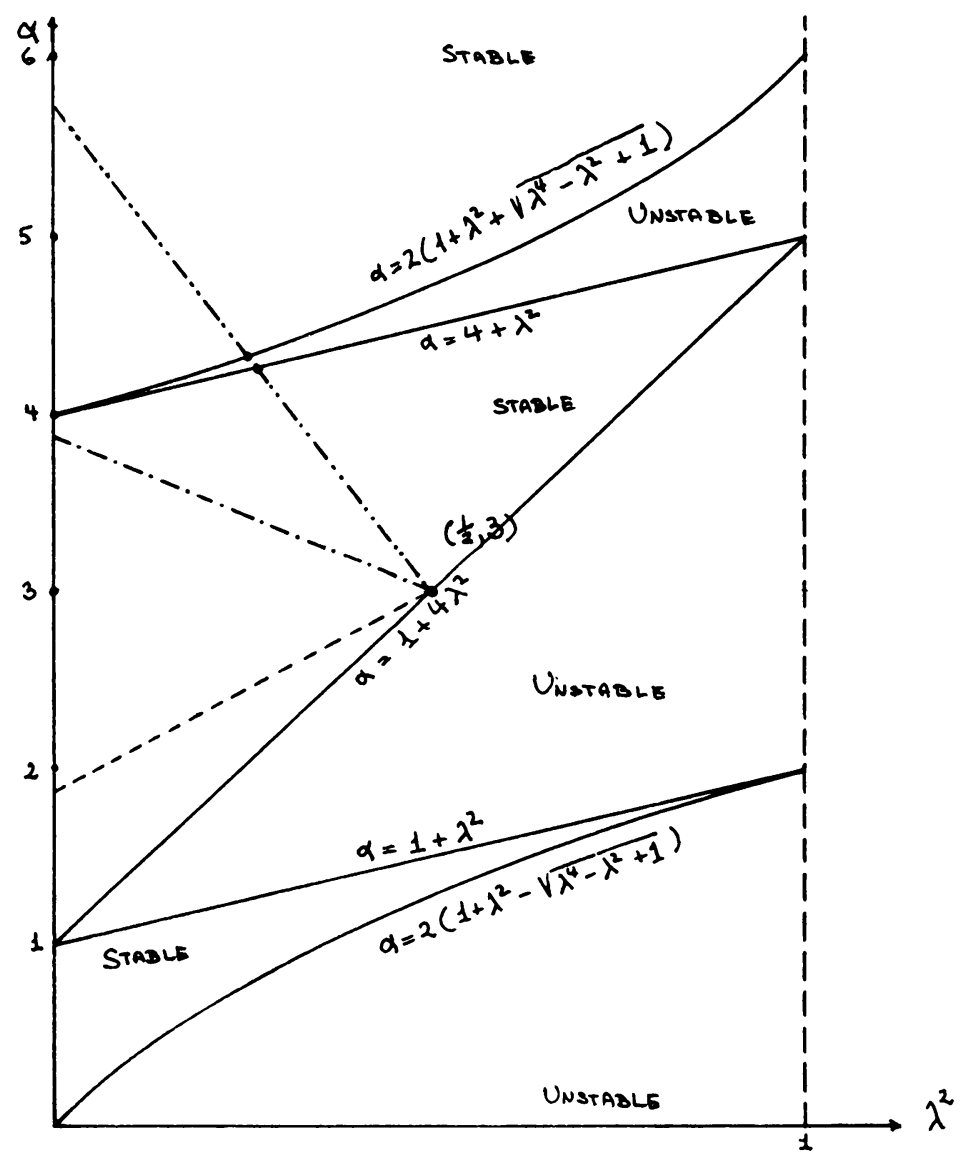

FIG. 2. 
corresponding line from $\lambda^{2}=0$ to $\lambda^{2}=\frac{1}{2}$, and the quantity $\hat{u}_{21}{ }^{2}(T)=\hat{u}_{21}{ }^{2}\left(T, \alpha, \lambda^{2}\right)$ regarded as a function of $\epsilon h$ takes on values less than or greater than 1 according as $\left(\alpha, \lambda^{2}\right)$ is passing through a stable or unstable region.

5. Proof of the theorem. We fix values of $a_{2}, a_{1}, b_{1}$ and, consequently, a line $\alpha=$ $4 \lambda^{2}\left(1-\left(a_{2} / a_{1}\right)\right)+2\left(a_{2} / a_{1}\right)+1$. To prove the first part of the theorem, we note that if $0<$ $a_{2} / a_{1}<3 / 2$, then for all values of $\epsilon h,\left(\alpha, \lambda^{2}\right)$ lies in the second stability region. Hence for 0 $<\lambda^{2}<\frac{1}{2}, \hat{u}_{21}{ }^{2}(T)<1$. Then the eignevalues will have non-zero imaginary part and $\omega_{1}$ will be elliptic except with $\hat{u}_{21}(T)=0$, where it is parabolic with eigenvalues $-1,-1$. When $a_{2} / a_{1}=\frac{3}{2}$, the only change is that at $\epsilon h=0, \omega_{1}$ is also parabolic with eigenvalues 1,1 . When $a_{2}=0$ (which corresponds to purely nonlinear coupling in Fig. 1) we have $\hat{u}_{21}(T) \equiv-1$ and $\omega_{1}$ is parabolic for all $\epsilon h$ with eigenvalues 1,1 .

When $a_{2} / a_{1}>\frac{3}{2}$ we have a non-empty range of hyperbolic values. The transition to hyperbolicity occurs when $4 \lambda^{2}\left(1-\left(a_{2} / a_{1}\right)\right)+2\left(a_{2} / a_{1}\right)+1=2\left[1+\lambda^{2}+\left(\lambda^{4}-\lambda^{2}+1\right)^{1 / 2}\right]$. Solving for $\lambda^{2}$ gives $\lambda^{2}=\frac{1}{2}-a_{1}\left(3 a_{2}\left(a_{2}-a_{1}\right)^{1 / 2} / 4 a_{2}\left(a_{2}-a_{1}\right)\right.$, and using formula (9) we find that the corresponding value of $\epsilon h$ and, hence of $k_{1}$, is $k_{1}=\left(4 a_{2}\left(a_{2}-a_{1}\right)-3 a_{1}^{2}\right) / 6 b_{1}$. In a similar way one finds that the transition from hyperbolicity to ellipticity is given by $k_{2}=$ $4 a_{2}\left(2 a_{2}-3 a_{1}\right) / 9 b_{1}$. For $0 \leq \epsilon h \leq k_{1}$ and $k_{2} \leq \epsilon h$ we can say that $\omega_{1}$ is elliptic except when $\hat{u}_{21}(T)=-1,0,1$ where it is parabolic.

Note: It will follow from the remarks below that parabolicity occurs at most on a set of measure 0 .

To prove the assertions concerning stability, we regard the eigenvalues $\Lambda_{ \pm}$as being obtained by the following composition of maps $=\Lambda_{ \pm}(\epsilon h)=f_{ \pm} \circ g_{\circ} l(\epsilon h)$. Here $l(\epsilon h)=\lambda^{2}(\epsilon h)$ is given by formula (9). The function $g$ is given by $g\left(\lambda^{2}\right)=\hat{u}_{21}(T)=\hat{u}_{21}\left(T\left(\lambda^{2}\right), \alpha, \lambda^{2}\right)$ with $\alpha=4 \lambda^{2}\left(1-\left(a_{2} / a_{1}\right)\right)+2\left(a_{2} / a_{1}\right)+1$ and the functions $f_{ \pm}$by $f_{ \pm}(u)=2 u^{2}-1 \pm\left(u^{2}\left(u^{2}-\right.\right.$ $1)^{1 / 2}$. We restrict the domain to $[0, k] \cup\left[k_{2}, \infty\right)$ so that only non-hyperbolic values are considered.

We now observe that $g$ is analytic in $\lambda^{2}$ for $0<\lambda^{2}<1$. To see this, note that since $\operatorname{sn}(u$, $\left.\lambda^{2}\right)$ is analytic in $\lambda^{2}$ for $0<\lambda^{2}<1$ [11, p. 493], the solutions of Eq. (12) are analytic in $\alpha$ and $\lambda^{2}$ as well as the independent variable for $0<\alpha, 0<\lambda^{2}<1$. Therefore the condition $\alpha$ $=4 \lambda^{2}\left(1-\left(a^{2} / a_{1}\right)\right)+2\left(a_{2} / a_{1}\right)+1$ implies that the solution $\hat{u}_{21}$ of Eq. (7) is analytic in $\lambda^{2}$ for $0<\lambda^{2}<1$. Now $T\left(\lambda^{2}\right)$ is also analytic in $\lambda^{2}$; indeed,

$$
T\left(\lambda^{2}\right)=\pi\left(1+\sum_{n=1}^{\infty} a_{n}{ }^{2} \lambda^{2 n}\right) \quad \text { with } \quad a_{n}=\frac{(2 n-1) \cdots 3 \cdot 1}{(2 n) \cdots 4 \cdot 2}
$$

provided $\lambda^{2}<1$. Thus $g$ is analytic in $\lambda^{2}$ for $0<\lambda^{2}<1$ and, a fortiori, for $0<\lambda^{2}<\frac{1}{2}$.

Referring now to the Arnold-Moser-Rüssman criterion stated in the introduction, let $F$ denote the complement of $H$ in the unit circle $S$. In particular, $F$ contains all roots of unity (and therefore will include the parabolic values of $\Lambda_{ \pm}$). Since $F$ has measure $0, f_{ \pm}^{-1}(F)$ has measure 0 in $[-1,1]$. This is easy to check using the monotonicity and differentiability properties of $f_{ \pm}$(see Appendix 2). Now the map $g$ is analytic on its domain and maps into $[-1,1]$. The analyticity can be used (Appendix 2) to show that $g^{-1}\left(f_{ \pm}^{-1}(F)\right.$ ) must have measure 0 . Finally the monotoneity of $l$ and the fact that its derivative is bounded away from 0 on compact sets can be used to show that the set $E \equiv l_{ \pm}^{-1}\left(g^{-1}\left(f^{-1}(F)\right)\right)$ has measure 0 in the set $D \equiv[0, k] \cup\left[k_{2}, \infty\right)$. This finishes the proof of the theorem.

6. The out-of-phase solution. The development here follows that of Secs. 2, 3 and 4, so we will omit many details. Each component of the out-of-phase solution $\omega_{2}$ will have 
Hamiltonian

$$
\hat{H}=\frac{1}{2} y^{2}+\frac{1}{2}\left(a_{1}+2 a_{2}\right) x^{2}+\frac{\epsilon}{4}\left(b_{1}+8 b_{2}\right) x^{4} .
$$

We again obtain Duffing's equation $\ddot{x}+A x+B x^{3}=0$ with $A=a_{1}+2 a_{2}, B=\epsilon\left(b_{1}+\right.$ $8 b_{2}$ ), which has the same solution as (2), namely (3), with $a, b$ replaced by $A, B$.

The Hessian evaluated along $\omega_{2}$ is

$$
H_{* *}\left(\omega_{2}\right)=\left[\begin{array}{cccc}
a_{1}+a_{2}+3 \epsilon\left(b_{1}+4 b_{2}\right) x^{2} & -a_{2}-12 \epsilon b_{2} x^{2} & 0 & 0 \\
-a_{2}-12 \epsilon b_{2} x^{2} & a_{1}+a_{2}+3 \epsilon\left(b_{1}+4 b_{2}\right) x^{2} & 0 & 0 \\
0 & 0 & 1 & 0 \\
0 & 0 & 0 & 1
\end{array}\right] \text {. }
$$

We obtain

$$
\ddot{z}_{1 j}=-\alpha z_{1 j}-\beta z_{2 j}, \quad \ddot{z}_{2 j}=-\beta z_{1 j}-\alpha z_{2 j}
$$

with $\alpha=a_{1}+a_{2}+3 \epsilon\left(b_{1}+4 b_{2}\right) x^{2}, \beta=-a_{2}-12 \epsilon b_{2} x^{2}$. The system is uncoupled by introducing

$$
u_{1 j}=z_{1 j}+z_{2 j}, \quad u_{2 j}=z_{1 j}-z_{2 j},
$$

and the $u_{1 j}$ satisfy the analogues of Eqs. 4 and 5:

$$
\begin{gathered}
\ddot{u}_{1 j}=-\left(a_{1}+3 \epsilon b_{1} x^{2}\right) u_{1 j}, \\
\ddot{u}_{2 j}=-\left(a_{1}+2 a_{2}+3 \epsilon\left(b_{1}+8 b_{2} x^{2}\right) u_{2 j},\right.
\end{gathered}
$$

with appropriate initial conditions.

We transform these into proper Lamé equations obtaining in place of (6) and (7) the following:

$$
\begin{gathered}
\hat{u}_{1 j}{ }^{\prime \prime}+\left[4 \lambda^{2}+1-2 \frac{a_{2}+12 \epsilon b_{2} x_{0}{ }^{2}}{A+B x_{0}{ }^{2}}-\frac{b_{1}}{b_{1}+8 b_{2}} \cdot 3 \cdot 2 \lambda^{2} s n^{2}\left(u, \lambda^{2}\right)\right] \hat{u}_{1 j}=0, \\
\hat{u}_{2 j}{ }^{\prime \prime}+\left[4 \lambda^{2}+1-3 \cdot 2 \lambda^{2} \operatorname{sn}^{2}\left(u, \lambda^{2}\right)\right] \hat{u}_{2 j}=\theta .
\end{gathered}
$$

Now, as in Sec. 3, we find that $\Lambda_{ \pm}=\frac{1}{2}\left[D-2 \pm(D(D-4))^{1 / 2}\right]$ where $D=\operatorname{Tr} Z(\tau)$. Moreover, as was the case in Sec. 3, the formula for $\operatorname{Tr} Z(\tau)$ is simplified by virtue of the fact that the terms from Eq. (15) drop out. Thus the eigenvalues are governed by the formula

$$
\operatorname{Tr} Z(\tau)=4 \hat{u}_{11}^{2}(T)
$$

Note: It can be shown that for a wide class of Hamiltonian systems the uncoupling via a linear transformation and the reduction of $Z(\tau)$ in terms of one Lamé equation (more generally a Hill's equation) can always be achieved. Moreover, similar results apply to systems with more than two degrees of freedom.

At this point we observe that Eq. (14) is a much more difficult Lamé equation than was Eq. (7) because of the factor $b_{1} /\left(b_{1}+8 b_{2}\right)$ in the non-autonomous part. It is easy to show (and is part of a well-known general result) via the transformation $\xi=\operatorname{am}\left(u, \lambda^{2}\right)$ and an application of Ince's theorem [6, p. 93] that no regions of instability for Eq. (14) collapse unless $b_{2}=0$ or $b_{1}=4 b_{2}$. We can construct stability charts in these two special cases and we shall do so to illustrate the rather different stability behavior possible for the out-ofphase mode. 
Case 1: $b_{2}=0$. In this case (14) has the form (12) so that the same stability chart may be used. Here, however, the lines determined by (14) are given by

$$
\alpha=4 \lambda^{2}\left(1+\frac{a_{2}}{a_{1}+2 a_{2}}\right)+\frac{a_{1}}{a_{1}+2 a_{2}},
$$

and the configuration is that shown in Fig. 3.

We see that when $0<a_{1} /\left(a_{1}+2 a_{2}\right)<1, \omega_{1}$ will be elliptic stable, except on a set of measure 0 , until $\alpha=1+\lambda^{2}$, which translates to $\epsilon h=\left(12 a_{2}\left(a_{1}+2 a_{2}\right)+8\right) / 9 b_{1}$, when it becomes and remains hyperbolic. When $a_{1} /\left(a_{1}+2 a_{2}\right)=1$, the solution is parabolic for all $\epsilon h$.

Case 2: $b_{1}=4 b_{2}$. Here a new stability chart is required since Eq. (14) has the form

$$
y^{\prime \prime}+\left(\alpha-2 \lambda^{2} s n^{2}\left(u, \lambda^{2}\right)\right) y=0
$$

with $\alpha=4 \lambda^{2}\left(a_{2} /\left(a_{1}+2 a_{2}\right)+a_{1} /\left(a_{1}+2 a_{2}\right)\right.$.

One has the following solutions of (16):

$$
\begin{aligned}
& y=d n\left(u, \lambda^{2}\right) \text { when } \alpha=\lambda^{2} \quad \text { period } T\left(\lambda^{2}\right), \\
& y=\operatorname{cn}\left(u, \lambda^{2}\right) \text { when } \alpha=1 \quad \text { period } 2 T\left(\lambda^{2}\right), \\
& y=\operatorname{sn}\left(u, \lambda^{2}\right) \text { when } \alpha=\lambda^{2}+1 \text { period } 2 T\left(\lambda^{2}\right) .
\end{aligned}
$$

Since Eq. (16) has exactly two nondegenerate instability regions we obtain the chart in Fig. 4. Here, for $0<a_{1} /\left(a_{1}+2 a_{2}\right)<1, \omega_{2}$ is elliptic stable for all values of $\epsilon h$ outside a set of measure 0 . For $a_{1} /\left(a_{1}+2 a_{2}\right)=1, \omega_{2}$ is parabolic for all $\epsilon h$.

Further study of the out-of-phase mode, if it were to based on the techniques of this paper, would require stability charts for equations of the form (14) with $b_{1} /\left(b_{1}+8 b_{2}\right)$ as

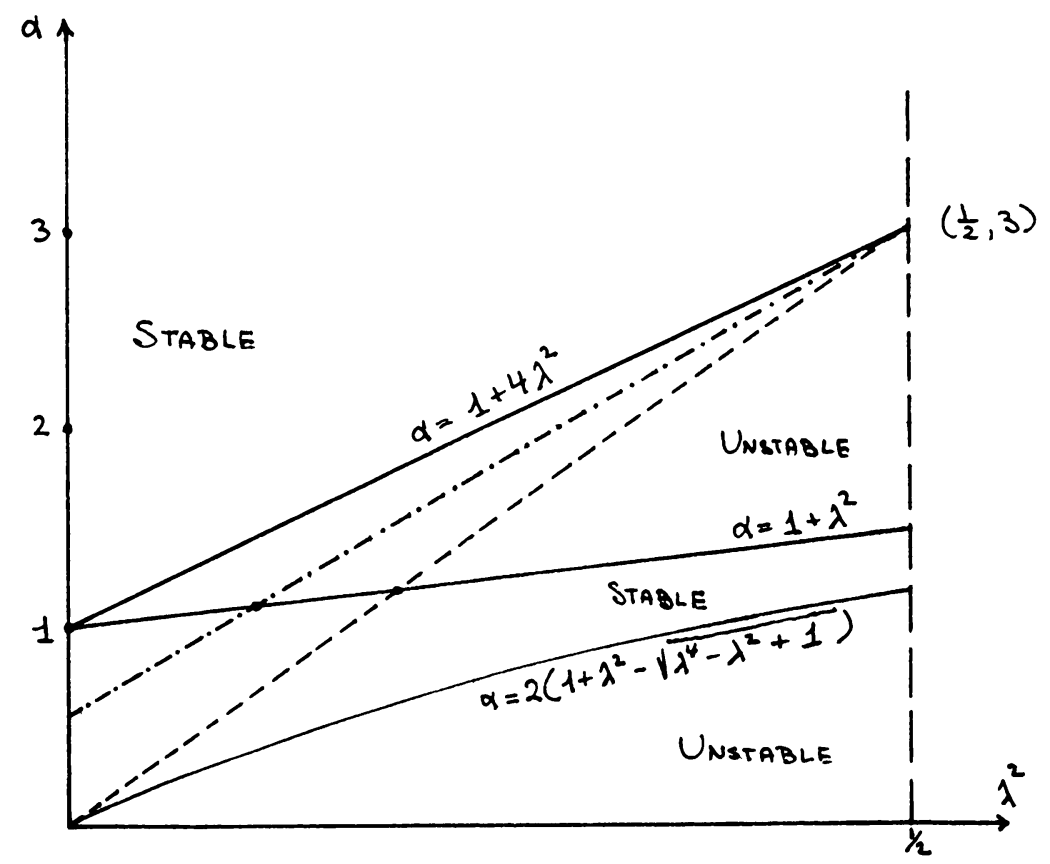

FIG. 3. 


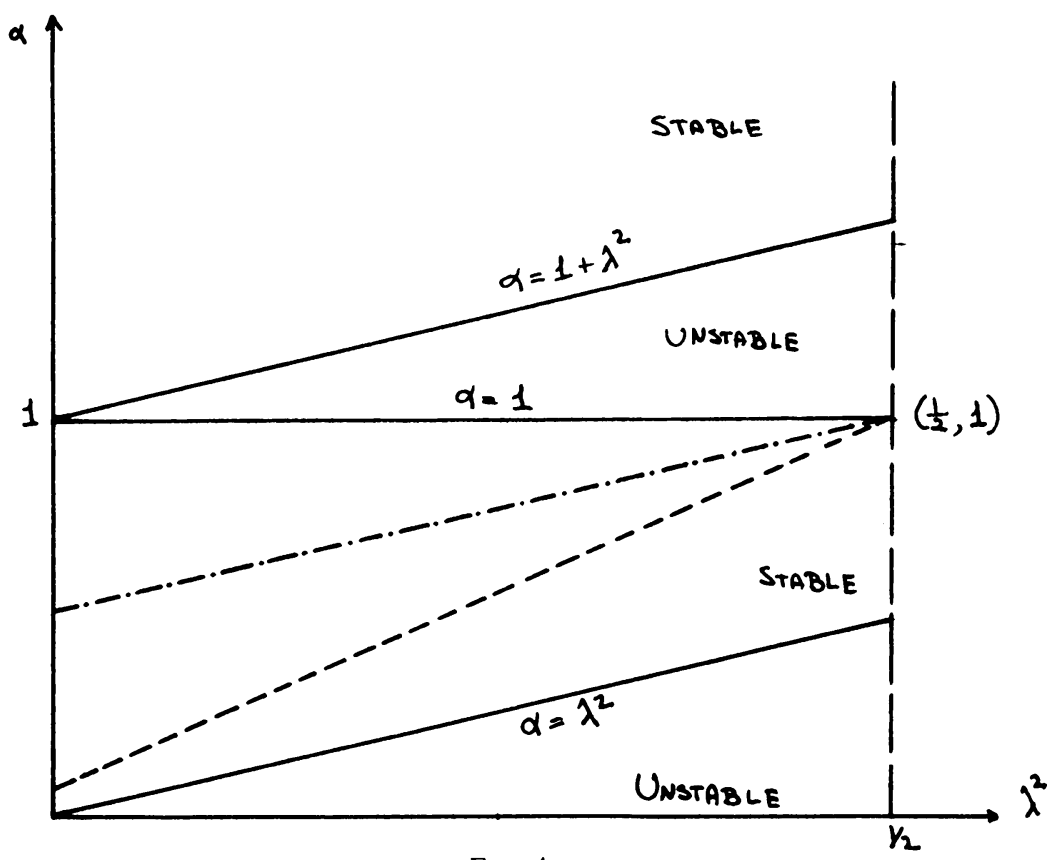

FIG. 4.

parameter. This seems difficult. We also mention that if one wishes to consider the stability problem for Hamiltonians with more general nonlinear terms, e.g. high even powers rather than fourth powers in (1), then one is lead to more general Hill's equations in place of (12). Here again not enough is known about the stability charts of the equations to obtain satisfying results.

Appendix 1. In this appendix we show that the complement of the set $H$ defined in the introduction in connection with the Arnold-Moser-Rüssmann criterion has measure 0 in the unit circle $S$. Denoting the complement by $G$, we see that $G$ can be written as the intersection of sets $G(k), k=1,2, \cdots$, where $G(k)=\left\{\mu \in S \mid\right.$ for some $n,\left|\mu^{n}-1\right| \leq$ $\left.k^{-1} n^{-k}\right\}$. Since $G(1) \supset G(2) \supset \cdots$, it suffices to prove that the measure of $G(k)$ tends to 0 as $k \rightarrow+\infty$. Write $G(k)=\bigcup_{n=1}^{\infty} G(k, n)$, where $G(k, n)=\left\{\mu \in S|| \mu^{n}-1 \mid \leq k^{-1} n^{-k}\right\}$. Letting $r_{n}=k^{-1} n^{-k}$ and choosing $k$ sufficiently large, we observe that $G(k, n)$ consists of $n$ disjoint arcs in $S$ centered at the $n$th roots of unity, each having arc length equal to $(4 / n)$ $\sin ^{-1}\left(r_{n} / 2\right)$. Thus the measure of $G(k, n)$ is just $4 \sin ^{-1} r_{n} / 2$ and the measure of $G(k)$ is at most $4 \sum_{n=1}^{\infty} \sin ^{-1} r_{n} / 2$. Now letting $\theta_{n}=4 \sin ^{-1} r_{n} / 2$ and choosing $k$ large enough so that $\theta_{n} / 4<1$, we have

$$
r_{n} / 2=\sin \theta_{n} / 4=\left(\theta_{n} / 4\right)-\left(\theta_{n} / 4\right)^{3} q
$$

where $0<q<1 / 3$ !, so that $r_{n} / 2 \geq 5 \theta_{n} / 24$ or $\theta_{n} \leq(12 / 5) r_{n}=12 / 5 k^{-1} n^{-k}$. Hence the measure of $G(k, n)$ is at most $\sum_{n=1}^{\infty}(12 / 5) k^{-1} n^{-k}$. For $k \geq 2$, the series converges with sum less than $12 / 5(k-1)$.

Appendix 2. In this appendix we verify the assertions of Sec. 5 concerning the preimage of the set $F$ under the composition $\Lambda_{ \pm}=f_{ \pm} \circ g_{\circ} l$.

Thus, let $F$ have measure zero in the unit circle $S$ and consider, first, one of the maps 
$f_{ \pm}$, say $f_{+}:[-1,1] \rightarrow S$ given by $f_{+}(u)=2 u^{2}-1+\left(u^{2}\left(u^{2}-1\right)\right)^{1 / 2}$. Let $I=[-1,0]$ so that $f \mid I$ is one-to-one. It suffices to show that the measure of $f_{+}{ }^{-1}(F) \cap I$ is zero. Let $K$ be the compact subset of $S$ defined by $K=f_{+}([-1+\epsilon / 4, \epsilon / 4])$ and let $k>0$ be a lower bound for $\left|f^{\prime}(u)\right|$ on the interval $L \equiv[-1+\epsilon / 8,-\epsilon / 8] \supset K$. Let $\left\{W_{i} \mid i=1,2, \cdots\right\}$ be a denumerable cover of the set $F \cap K$ with open sets each of which lies in $f(L)$ and such that the measure of $\cup W_{i}$ is less than $\epsilon k / 2$. Then, for each $i, f_{+}{ }^{-1}\left(W_{i}\right)=V_{i}=\left(a_{i}, b_{i}\right)$ lies in $L$ and the measure of $W_{i}$ can be written:

$$
m\left(W_{i}\right)=\int_{a_{l}}^{b_{i}}\left|f^{\prime}(u)\right| d u \geq\left(b_{i}-a_{i}\right) k=k m\left(V_{i}\right) .
$$

Now $f_{+}{ }^{-1}(F) \subset \cup f_{+}^{-1}\left(W_{l}\right) \cup(I-K)$, so that

$$
m\left(f_{+}^{-1}(F)\right) \leq \sum \frac{1}{k} m\left(W_{i}\right)+\epsilon / 4+\epsilon / 4 \leq \epsilon,
$$

as desired.

Next we consider the map $g:(0,1) \rightarrow[-1,1]$ of Sec. 5 . Recall that $g$ is real analytic on $(0,1)$ and therefore $\left\{\lambda^{2} \mid g^{\prime}\left(\lambda^{2}\right)=0\right\}$ is a countable set with no accumulation points in $(0$, 1). Therefore we may write $(0,1)$ as the (possibly infinite) union of closed intervals $\left\{I_{i} i=1\right.$, $2, \cdots\}$ such that $g^{\prime}$ does not vanish on the interior of $I_{i}$. The proof of the preceding paragraph may now be applied to each $I_{i}$ to show that the measure of $g^{-1}\left(f_{ \pm}^{-1}(F)\right) \cap I_{i}$ is zero for each $i$.

Finally, the map $l:\left[0, k_{1}\right] \cup\left[k_{2}, \infty\right) \rightarrow\left[0, \frac{1}{2}\right)$ given by $l(r)=\frac{1}{2}-\left(a_{1} /\left(a_{1}{ }^{2}+2 r b_{1}\right)^{1 / 2}\right)$ is monotone and the derivative is bounded away from 0 on compact sets. Thus a variation on the same argument shows that $l^{-1}\left(g^{-1}\left(f_{ \pm}^{-1}(F)\right)\right)$ will have measure zero on $\left[0, k_{1}\right] \cup\left[k_{2}\right.$, $\infty)$.

\section{REFERENCES}

[1] V. I. Arnold, Kleine Nenner und Stabilitätsprobleme der klassischen Mechanik und der Himmelsmechanik, Uspekhi Mat. Nauk USSR 18, 6, 91-192 (1963)

[2] F. M. Arscott, Periodic differential equations, Pergamon, 1964

[3] R. Churchill, H. Jurenka and E. Thomas, A note on iso-energetic stability, Int. J. Non-linear Mech. 12 , 37-43 (1977)

[4] R. Churchill, G. Pecelli, D. Rod, and S. Sakolic, Coexistence of stable and random motion, Rocky Mountain J. Math. 7, 445-456 (1977).

[5] N. W. McLachlan, Ordinary non-linear differential equations in engineering and physical sciences, $2 \mathrm{nd}$ edition, Clarendon Press, 1956

[6] W. Magnus and S. Winkler, Hill's equation, Interscience Publishers, 1966

[7] J. K. Moser, On invariant curves of area-preserving mappings of an annulus, Nachr. Akad. Wiss. Göttingen Math.-Phys. KI. II, 1 (1962)

[8] J. G. Proctor and R. A. Struble, Motion of two weakly coupled nonlinear oscillators, Arch. Rat. Mech. A nal. 18, 293-303 (1965)

[9] R. M. Rosenberg and C. P. Atkinson, On the natural modes and their stability in non-linear two-degree-offreedom systems, J. Appl. Mech. 26, 377-385 (1959)

[10] H. Rüssmann, Über die Normalform analytischer Hamiltonscher Differentialgleichungen in der Nähe einer Gleichgewichtslösung, Math. Annalen 169, 55-72 (1967)

[11] E. T. Whittaker and G. N. Watson, A course of modern analysis, 4th edition, Cambridge University Press, 1952 